\title{
Mitral degenerative valve leaflets suspected as primary valvular tumor: a case report
}

\author{
Shiqiang Wang ${ }^{1}$, Huaidong Chen ${ }^{1}$, Ximing Qian ${ }^{1}$, and Fan $\mathrm{He}^{1}$ \\ ${ }^{1}$ Department of Cardiac Surgery Sir Run Run Shaw Hospital School of Medicine Zhejiang \\ University Hangzhou China
}

November 22, 2021

\begin{abstract}
Introduction: Echocardiography plays an important role in the diagnosis of heart disease. However, sometimes misdiagnosis information is also provided. Methods and Results: We report a rare case of preoperative echocardiography misdiagnosed as a primary mitral valvular tumor with severe regurgitation. During the surgery procedure, the true lesion was found to be mitral valve leaflet prolapse due to degenerative mitral valve disease. Conclusions: For individual patient, it may need to combine clinical symptoms and wide use of echocardiography and CT or MRI to make the optimal clinical decision.
\end{abstract}

\section{Introduction}

Primary valvular tumors and valve degeneration are generally easy to distinguish, but in some special cases, the two have a certain similarity in echocardiography, especially for patients with atypical symptoms, it is often misdiagnosed one for another. Here, a case of mitral valve degenerative disease suspected to be diagnosed as primary valvular tumor was revealed.

\section{Case Report}

Due to recurrent chest tightness and shortness of breath, the patient came to the emergency department of our hospital. Transthoracic echocardiography revealed mitral leaflet cystic nodules, suspected benign tumor or infective endocarditis with vegetations, and mitral valve with severe regurgitation. The patient had no other comorbidities except hypertension. Systolic murmur in apical region was heard. Transesophageal echocardiography revealed the presence of multiple cystic structures with significant separation in the A1, $\mathrm{C} 1$, and $\mathrm{P} 1$ leaflets of the mitral Carpentier nomenclature ${ }^{1}$. The larger one was located in the P1 area, about $18.3^{*} 14.2 \mathrm{~mm}$ in size, and smaller in the A1 area about $9.9^{*} 5.4 \mathrm{~mm}$ (Figure 1 ). The conclusion of ultrasonography showed that primary valve tumor should be considered first after excluding infective endocarditis. We had done a lot of examinations related to infective endocarditis, such as cranial Computed Tomography (CT), brain magnetic resonance imaging (MRI) and blood culture, etc. But our case did not fulfill the modified Duke criteria for the clinical diagnosis of infective endocarditis. Then we planned to perform valve tumor resection and mitral valve repair. However, no tumors were occupied and no infectious vegetations were found on the valve leaflets during the operation. The cystic structure described by ultrasound was caused by prolapse due to leaflet degeneration (Figure 2). In order to maintain the integrity of the leaflet structure, we performed folding repair for the mitral leaflet. The patient was recovered well after operation, and the result of echocardiography evaluation was satisfactory.

\section{Discussion}

Echocardiography has evolved rapidly since its advent in the 1950s and is now the modality of choice for imaging occupying cardiac lesions. It provides high-quality, real-time images, which are invaluable in the 
evaluation of cardiac masses. Although transthoracic echocardiography is an excellent initial diagnostic technique for the evaluation and diagnosis of cardiac masses, transesophageal echocardiography provides superior image resolution and better visualization of cardiac masses in patients with poorly studied transthoracic echocardiography ${ }^{2}$. For some diseases with quite similar echocardiographic presentation need to differential diagnosis carefully, especially for cases where the symptomatic presentation cannot be clearly identified ${ }^{3}$. The treatment options differ greatly between the two groups of potentially confusing diseases, such as the primary valve tumors and valve degeneration in this case.

Primary cardiac tumors are fairly uncommon, with an average incidence rate about $0.02 \%$, while primary valvular tumors account for only $10 \%$ among them. Among the more common types are myxoma, papillary fibroelastoma, and lipoma ${ }^{4,5}$. The features of echocardiogram are often pedunculate, often a solitary mass, usually at the mid-portion of valve leaflets, and with a frond-like characteristic surface ${ }^{6}$. The primary valvular tumors need to be surgically removed if there is mobility, even for asymptomatic patients, because of the potential cerebral and cardiac embolization ${ }^{7,8}$. Valvular degenerative disease due to the absence of fibrin in the valve leads to lengthy chordae tendineae and leaflet prolapse, which often causes different degrees of valvular regurgitation ${ }^{9,10}$. In this case, the apex systolic murmur was consistent with the degree of echocardiographic mitral valve regurgitation.

Generally, only patients with severe mitral regurgitation combined with symptoms of cardiac insufficiency require surgery. This patient had severe mitral regurgitation before operation, and the wrong judgment before operation did not cause any adverse effect. However, for suspected valve masses without valve regurgitation, more accurate evaluation and identification of primary valvular tumors and valve degenerative lesions is clearly warranted. For individual patient, it may need to combine clinical symptoms and wide use of echocardiography and CT or MRI to make the optimal clinical decision.

\section{Consent for publication}

Informed written consent was obtained from the patient for publication of this case and accompanying images.

\section{The authors have no conflicts of interests to disclose.}

\section{Author contributions:}

Writing-original draft: Shiqiang Wang;

Writing-review \& editing: Huaidong Chen; Ximing Qian;

Conceptualization and Supervision: Fan He;

\section{Reference}

1. Carpentier A, Relland J, Deloche A, et al. Conservative management of the prolapsed mitral valve. Ann Thorac Surg. 1978;26(4):294-302.

2. Ragland MM, Tak T. The role of echocardiography in diagnosing space-occupying lesions of the heart.Clin Med Res. 2006;4(1):22-32.

3. Auriti A, Chieffi M, Cianfrocca C, et al. Giant vegetation of the mitral valve simulating primary cardiac tumor. Echocardiography. 2004;21(2):183-185.

4. Georghiou GP, Vidne BA, Sahar G, Sharoni E, Fuks A, Porat E. Primary cardiac valve tumors. Asian cardiovascular \& thoracic annals. 2010;18(3):226-228.

5. Issa OM, Reyna J, Santana O, DeBeer S, LaPietra A, Lamelas J. Cardiac angiofibroma: a rare primary cardiac tumor. J Card Surg. 2013;28(4):404-405.

6. Prifti E, Ikonomi M, Veshti A, Demiraj A, Xhaxho R. Papillary fibroelastoma of the anterior leaflet of the mitral valve mimicking vegetation. Int J Surg Case Rep.2015;14:19-22. 
7. Pacini D, Farneti PA, Leone O, Galli R. Cardiac papillary fibroelastoma of the mitral valve chordae.Eur J Cardiothorac Surg. 1998;13(3):322-324.

8. Gowda RM, Khan IA, Nair CK, Mehta NJ, Vasavada BC, Sacchi TJ. Cardiac papillary fibroelastoma: a comprehensive analysis of 725 cases. Am Heart J.2003;146(3):404-410.

9. Del Forno B, Ascione G, De Bonis M. Advances in Mitral Valve Repair for Degenerative Mitral Regurgitation: Philosophy, Technical Details, and Long-Term Results. Cardiology clinics. 2021;39(2):175-184.

10. Michelena HI, Topilsky Y, Suri R, Enriquez-Sarano M. Degenerative mitral valve regurgitation: understanding basic concepts and new developments. Postgraduate medicine. 2011;123(2):56-69.

\section{Hosted file}

Figure.docx available at https://authorea.com/users/426057/articles/546427-mitraldegenerative-valve-leaflets-suspected-as-primary-valvular-tumor-a-case-report 